ORIGINAL ARTICLE

\title{
MYC is a positive regulator of choline metabolism and impedes mitophagy-dependent necroptosis in diffuse large B-cell lymphoma
}

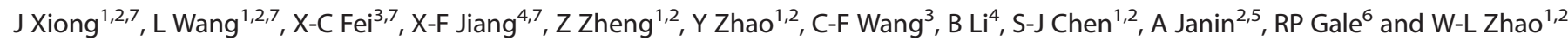

The activation of oncogenes can reprogram tumor cell metabolism. Here, in diffuse large B-cell lymphoma (DLBCL), serum metabolomic analysis revealed that oncogenic MYC could induce aberrant choline metabolism by transcriptionally activating the key enzyme phosphate cytidylyltransferase 1 choline-a (PCYT1A). In B-lymphoma cells, as a consequence of PCYT1A upregulation, MYC impeded lymphoma cells undergo a mitophagy-dependent necroptosis. In DLBCL patients, overexpression of PCYT1A was in parallel with an increase in tumor MYC, as well as a decrease in serum choline metabolite phosphatidylcholine levels and an International Prognostic Index, indicating intermediate-high or high risk. Both in vitro and in vivo, lipid-lowering alkaloid berberine (BBR) exhibited an anti-lymphoma activity through inhibiting MYC-driven downstream PCYT1A expression and inducing mitophagy-dependent necroptosis. Collectively, PCYT1A was upregulated by MYC, which resulted in the induction of aberrant choline metabolism and the inhibition of B-lymphoma cell necroptosis. Referred as a biomarker for DLBCL progression, PCYT1A can be targeted by BBR, providing a potential lipid-modifying strategy in treating MYC-High lymphoma.

Blood Cancer Journal (2017) 7, e582; doi:10.1038/bcj.2017.61; published online 7 July 2017

\section{INTRODUCTION}

Diffuse large B-cell lymphoma (DLBCL) is one of the most common subtype of non-Hodgkin lymphoma with considerable clinical and biological heterogeneity. ${ }^{1}$ Although a durable complete remission can be achieved by using rituximab combined with chemotherapy in a substantial proportion of patients, up to $30 \sim 40 \%$ of DLBCL cases are either relapsed or refractory to current standard treatment. ${ }^{2}$ Therefore, the identification of actionable biomarkers will be helpful in improving the clinical outcome of high-risk DLBCL patients. ${ }^{3}$

Initially uncovered as the target of $\mathrm{t}(8 ; 14)(\mathrm{q} 24 ; \mathrm{q} 32)$ chromosome translocation in Burkitt lymphoma, ${ }^{4}$ MYC is a master regulator in DLBCL pathogenesis ${ }^{5}$ and renders lymphoma cell resistance to chemotherapy. ${ }^{6}$ Clinically, MYC overexpression is related to increased risk of disease relapse and indicates poor disease outcome in DLBCL patients. ${ }^{7}$ As direct targeting of MYC appears difficult, ${ }^{8}$ alternative therapeutic strategies to specifically modulate MYC-driven downstream effectors warrants further investigation. ${ }^{9,10}$

Correlating with genomics, transcriptomics and proteomics, metabolomics is the end-point of 'multi-omics' cascades and provides a 'real-world' assessment of cancer cell physiology. ${ }^{11}$ Generally involved in genetic transcription, MYC alters multiple tumor metabolic processes such as glycolysis, nucleotide and lipid synthesis. $^{12}$ Previous works indicated that MYC regulates lipid metabolism during lymphomagenesis. ${ }^{13}$ It has recently been reported that, as an indispensible component of lipid synthesis, choline metabolism is dysregulated in lymphoma. ${ }^{14}$ However, the exact relationship between choline metabolism and lymphomagenic MYC expression remains undetermined.

Lipid metabolism has been exploited as a promising target for cancer treatment. ${ }^{15}$ Berberine (BBR) is an alkaloid initially extracted from Chinese herbs and possesses multiple antimetabolism properties, in particular the lipid-lowering effects. ${ }^{16}$ Under a clinically achievable concentration, BBR is both effective and safe in treating hyperlipidemia patients via the modulation of lipid profile. ${ }^{17}$ Experimentally, BBR can inhibit tumor cell growth in hematological malignancies, inducing cell apoptosis and caspaseindependent cell death. ${ }^{18,19}$ Therefore, to determine whether BBR has therapeutic effect on lymphoma lipid metabolism is of considerable interest.

The present study showed that MYC dysregulates choline metabolism and impedes lymphoma cell necroptosis in a mitophagy-dependent manner by transcriptionally activating the key enzyme phosphate cytidylyltransferase 1 choline-a (PCYT1A). Through targeting PCYT1A expression, the lipidlowering alkaloid BBR inhibited the MYC-driven aberration of choline metabolism and induced lymphoma cell necroptosis, providing a potential lipid-modifying strategy in treating MYCHigh lymphoma.

\footnotetext{
'State Key Laboratory of Medical Genomics, Shanghai Institute of Hematology, Shanghai Rui Jin Hospital, Shanghai Jiao Tong University School of Medicine, Shanghai, China: ${ }^{2}$ Pôle de Recherches Sino-Français en Science du Vivant et Génomique, Laboratory of Molecular Pathology, Shanghai Rui Jin Hospital, Shanghai, China; ${ }^{3}$ Department of Pathology, Shanghai Rui Jin Hospital, Shanghai Jiao Tong University School of Medicine, Shanghai, China; ${ }^{4}$ Department of Nuclear Medicine, Shanghai Rui Jin Hospital, Shanghai Jiao Tong University School of Medicine, Shanghai, China; ${ }^{5}$ Laboratory of Pathology, Paris Diderot University, U1165 Inserm, Paris, France and ${ }^{6}$ Haematology Research Centre, Division of Experimental Medicine, Department of Medicine, Imperial College London, London, UK. Correspondence: Dr W-L Zhao, State Key Laboratory of Medical Genomics, Shanghai Institute of Hematology, Shanghai Rui Jin Hospital, Shanghai Jiao Tong University School of Medicine, 197 Rui Jin Er Road, Shanghai 200025, China. E-mail: zhao.weili@yahoo.com

${ }^{7}$ These authors contributed equally to this work.

Received 16 January 2017; revised 19 May 2017; accepted 1 June 2017
} 


\section{MATERIAL AND METHODS}

\section{Patients}

Metabolic profile was assessed on the serum samples of 80 de novo patients with $\mathrm{DLBCL}$, including the training set $(N=30)$ and the validation set $(N=50)$. Histological diagnoses were established according to the World Health Organization classification. ${ }^{20}$ Quantitative detection of metabolites was further performed on serum samples of 108 de novo DLBCL cases. All patients were treated by rituximab combined with chemotherapy (cyclophosphamide, doxorubicin, vincristine and prednisone). Patients clinical characteristics were listed in Supplementary Table S1. Written informed consents were obtained from patients in accordance with the Declaration of Helsinki. The study was approved by Shanghai Rui Jin Hospital Review Board.

\section{Cells and reagents}

DLBCL cell line DB with MYC overexpression and Burkitt lymphoma cell line Ramos with MYC translocation (American Type Culture Collection, Manassas, VA, USA) were cultured in RPMI-1640 medium supplemented with $10 \%$ heat-inactivated fetal bovine serum in a humidified atmosphere of $95 \%$ air and $5 \% \mathrm{CO}_{2}$ at $37^{\circ} \mathrm{C}$. HEK-293T cell was cultured in DMEM medium supplemented with $10 \%$ heat-inactivated fetal bovine serum. MYC inhibitor 10058-F4 and mitophagy inhibitor Mdivi-1 were obtained from Selleck (Houston, TX, USA). BBR was from Sigma-Aldrich (St Louis, MO, USA). Nucleic acid synthesis inhibitor Actinomycin was from Abcam (Cambridge, UK).

\section{Cell viability}

Cell $\left(5 \times 10^{5} / \mathrm{ml}\right)$ were seeded in 96 -well plates and incubated with indicated concentration of reagents. Cell growth was assessed by CCK8 (1:10, Dojindo, Kumamoto, Japan) and the absorbance was measured at $450 \mathrm{~nm}$ by spectrophotometry. The percentage of cell growth inhibition was calculated as treated or transfected cells divided by untreated or nontransfected cells.

\section{RNA extraction and quantitative real-time PCR}

Total mRNA was extracted using TRlzol reagent (Invitrogen, Shanghai, China). Complementary DNA was synthesized using PrimeScript RT Reagent Kit with gDNA Eraser (TaKaRa, Dalian, China). Quantitative realtime PCR was performed by SYBR Premix Ex TaqTM II (TaKaRa) and ABI ViiA 7 (Applied Biosystems, Bedford, MA, USA) with primers against PCYT1A (Forward: 5'-GCCAAGGTCAATGCAAGGAA-3', Reverse: 5'-AAACTCTCA CAGGTCGCTCA-3'), PLA2G4C (Forward: 5'-CAGGAGTGGGACTTGGCTAA-3', Reverse: $5^{\prime}$-TCTTCCACGGGCTTCTTCAT-3'). GAPDH (Forward: 5'-GCTCATT TCCTGGTATGACAAC-3', Reverse: 5'-CTGTGAGGAGGGGAGATTCA-3') was used as an endogenous control.

\section{Metabolomic assay}

Serum samples $(100 \mu \mathrm{l})$ were assessed by ultra performance liquid chromatography (UPLC) and quadruple/time-of-flight mass spectrometry. The mass spectrometer was operated in both positive and negative electrospray ionization (ESI+/ESI-) mode. The UPLC-quadruple/time-offlight mass spectrometry ESI+ and ESI - raw data were analyzed by the MarkerLynx Applications Manager version 4.1 (Waters Corp., Milford, MA, USA) and total ion chromatography was generated. To identify different metabolomic profile between MYC-high and MYC-low groups, multivariate analysis was performed using SIMCA-P software version 12.0 (Umetrics AB, Umeå, Sweden). The unsupervised principal component analysis and supervised orthogonal partial least squares-discriminant analysis (OPLSDA) models were constructed. Reliability of OPLS-DA models was validated by response permutation test. Quality controls were prepared by mixing the same volume of each sample and repeatedly injected during the assay to monitor instrumental stability and avoid systematic bias. As for cell metabolites, lymphoma cells $\left(1 \times 10^{7} / \mathrm{ml}\right)$ were extracted by freeze-thaw method and cell lysate $(100 \mu \mathrm{l})$ was used for UPLC-triple quadrupole mass spectrometry analysis. The detailed parameters were as previously reported. ${ }^{14}$

\section{Gene network and pathway analysis}

Human Genome U133 Plus 2.0 Array GeneChip microarray (Affymetrix, Santa Clara, CA, USA) was performed on frozen tumor samples of nine nonGCB DLBCL cases. Genes filtered by KEGG (Kyoto Encyclopedia of Genes and Genomes) pathways were subsequently hierarchical clustered using MEV v.4.8.1 (Dana-Farber Cancer Institute, Boston, MA, USA).

\section{Cell transfection}

Cells $\left(1 \times 10^{6} / \mathrm{ml}\right)$ were transfected with luciferase reporter GV238promoter (GeneChem, Shanghai, China), pRL-TK Vector (Promega, Madison, WI, USA), PCYT1A-overexpressing vector pCMV6-PCYT1A, MYCoverexpressing vector pCMV6-MYC or vehicle pCMV6-ct (Origene, Rockville, MD, USA) using SuperFect transfection reagent (Qiagen, Hilden, Germany). Cells were transfected with MYC small interfering RNA (siRNA) (Cell Signaling, Beverly, MA, USA), three PCYT1A siRNAs (siRNA1: 5'-rArCrCr UrArArGrGrArCrArGrCrUrArCrArArArGrGrArAAG-3', siRNA2: 5'-rArGrGrUrC rArArUrGrCrArArGrGrArArGrArGrGrArGrAAA-3', siRNA3: 5'-rCrGrUrGrArUrCr UrCrArArCrArGrGrGrArArArUrUrGrUCA-3') or negative control siRNA (Origene), three PCYT1A short hairpin RNAs (shRNAs) or negative control shRNA (Origene) using HiperFect transfection reagent (Qiagen) according to manufacturer's instruction.

\section{Western blotting}

Western blotting was performed as described previously. ${ }^{21}$ Anti-MYC monoclonal antibody (Abcam, 1:1000), anti-PCYT1A monoclonal antibody (Cell Signaling, 1:1000), anti-p-AKT monoclonal antibody (Cell Signaling, 1:1000), anti-p-ERK monoclonal antibody (Cell Signaling, 1:1000) and AntiCDK6 monoclonal antibody (Cell Signaling, 1:1000) were used. Anti-Actin antibody (Cell Signaling, 1:2000) was used to ensure equivalent loading of total protein.

\section{Co-immunoprecipitation assay}

Co-immunoprecipitation assay was performed with $5 \times 10^{7}$ cells using Pierce Co-Immunoprecipitation Kit (Thermo, Pierce, Rockford, IL, USA) according to the manufacturer's protocols. Anti-RIP1 monoclonal antibody (BD Pharmingen, San Diego, CA, USA, 1:1000), anti-MLKL monoclonal antibody (Cell Signaling, 1:1000) and anti-RIP3 monoclonal antibody (Cell Signaling, 1:1000) were used.

\section{Luciferase reporter assay}

Luciferase activities were detected by Centro XS3 LB960 Luminometer (Berthold, Bad Wildbad, Germany) and Dual-Luciferase Reporter Assay System (Promega, Beijing, China).

\section{Chromatin immunoprecipitation assay}

Chromatin immunoprecipitation assays were performed with $2 \times 10^{7}$ cells using EZ-ChIP Kit (Millipore, Billerica, MA, USA). PCR and quantitative real-time PCR were performed with primer against the promoter of the PCYT1A (Forward: 5'-AGTTGGACACGGGAGCAG, Reverse: CGGAGGATG CAGCGAAGA-3'). Antibody against RNA Polymerase II was referred as positive control. Nonspecific lgG was referred as negative control.

\section{Immunohistochemistry}

Immunohistochemistry was performed on $5 \mu \mathrm{m}$ paraffin sections using anti-MYC monoclonal antibody (1:200) and anti-PCYT1A monoclonal antibody (1:100, Abcam), as previously reported. ${ }^{21}$ Protein expression levels were scored based on percentage of stained cells. ${ }^{22}$

\section{Confocal microscopy}

Acetone-fixed cells were co-incubated with anti-mitochondria monoclonal antibody MTC (1:100, Abcam) and anti-LC3A/B monoclonal antibody (1:200, Cell Signaling) as primary antibody and diaminotriazinylaminofluoresceinlabeled donkey anti-rabbit/mouse-lgG antibody (1:400, Abcam) as secondary antibody. Nuclei were counterstained with 4',6-diamidino-2-phenylindole. Confocal laser-scanning microscopy was applied to observe the co-localization of fluorescent staining.

\section{Transmission electron microscopy}

Cells and tissue samples were fixed for $2 \mathrm{~h}$ in $2 \%$ glutaraldehyde/ $0.1 \mathrm{~m}$ phosphate-buffered saline $(\mathrm{pH} 7.3)$ at $4{ }^{\circ} \mathrm{C}$, rinsed with $0.1 \mathrm{M}$ phosphatebuffered saline twice for $10 \mathrm{~min}$ at $4{ }^{\circ} \mathrm{C}$, postfixed in $1 \%$ osmium tetroxide/ $0.1 \mathrm{M}$ phosphate-buffered saline for $2 \mathrm{~h}$ at $4{ }^{\circ} \mathrm{C}$, dehydrated in graded ethanol, saturated in graded Epikote (Hexion, Columbus, $\mathrm{OH}, \mathrm{USA}$ ) and 
a

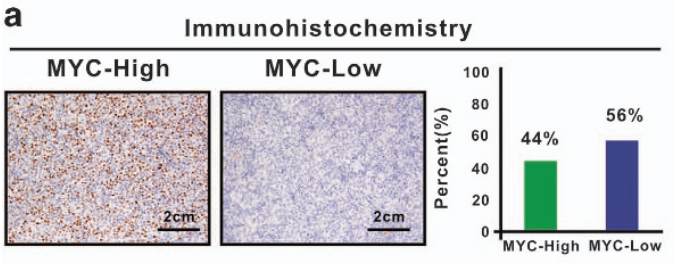

c

Pathways enriched with dysregulated metabolites

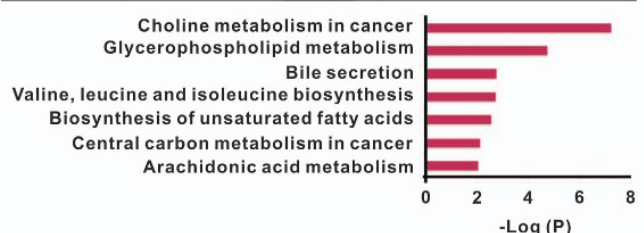

d

Gene expression profile of choline-related pathway

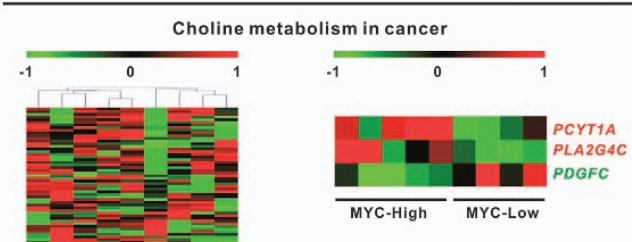

b
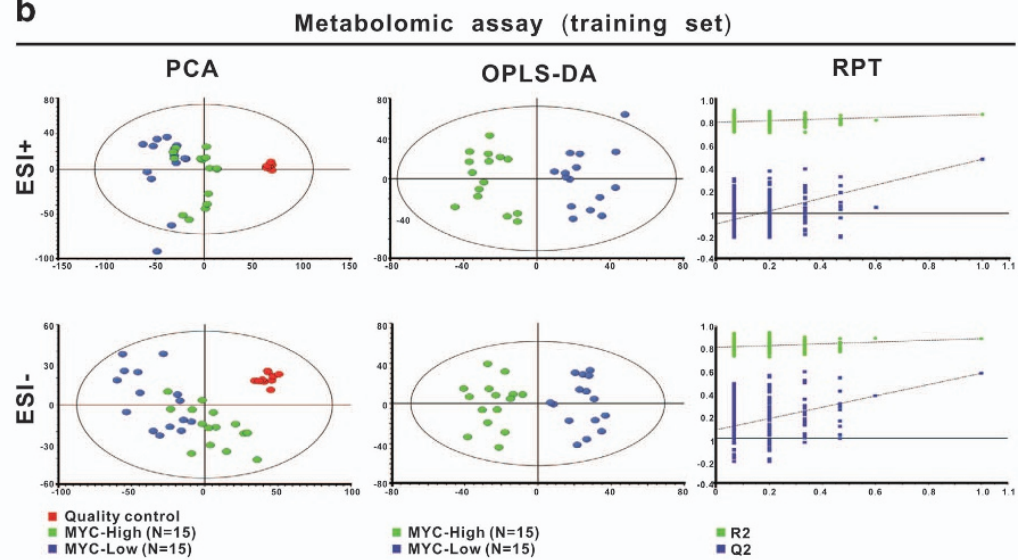

e Metabolomic assay (validation set)

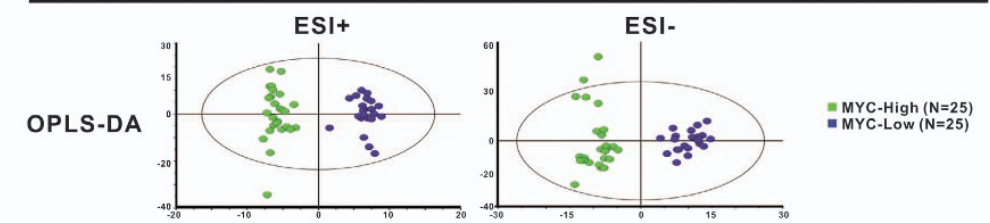

f

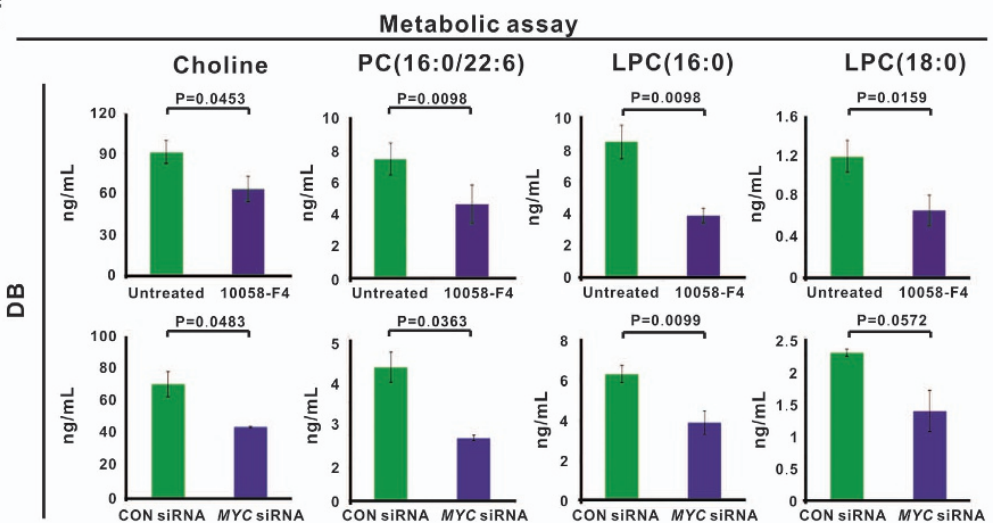

Figure 1. MYC was overexpressed and related to aberrant choline metabolism in DLBCL. (a) MYC protein was High ( $\geqslant 40 \%$ ) in 47 of 108 (44\%) patients with DLBCL. (b) The score plots of the principal component analysis (PCA) models (left panel) were set up using data from 15 MYC-High DLBCL cases (green plots), 15 MYC-Low DLBCL cases (blue plots) of the training set and 10 repeated quality controls (QCs, red plots). The score plots of the orthogonal partial least-squared discrimination analysis (OPLS-DA) models (middle panel) discriminated the MYC-High group (green plots) from the MYC-Low group (blue plots), which was confirmed by response permutation test (RPT, right panel). (c) Enrichment of KEGG pathways was performed on all the dysregulated metabolites using ConsensusPathDB. X-axis displayed $P$-values that were generated by $-\log (P)$ function. The $P<0.05$ and - $\log (P)>1.30$ were considered significant. (d) Genesets of choline metabolism in cancer and glycerophospholipid metabolism pathway were analyzed by gene network and pathway analysis on microarray data of nine non-GCB DLBCL cases according to MYC expression (left panel). Significantly dysregulated genes involved in the metabolic pathways were shown in right panel. Shades of red and green represented high or low expression. (e) The OPLS-DA score plots confirmed a global metabolic difference between the MYC-High group ( $N=25$, green plots) and the MYC-Low group $(N=25$, blue plots) in the validation set. (f) B-lymphoma cell line DB was either treated with 10058-F4 (40 $\mu \mathrm{m})$ for $48 \mathrm{~h}$ or transfected with MYC siRNA. Expression of Choline, PC(16:0/22:6), LPC(16:0) and LPC(18:0) were detected by UPLC-triple quadrupole mass spectrometry. Data in $\mathbf{f}$ were represented as mean \pm s.e.m. Assay in $\mathbf{f}$ was set up in triplicate.

embedded in Epon 812 (TAAB Laboratories, Reading, UK). Ultrathin sections were prepared with LKB V ultra microtome (LKB, Bromma, Sweden), stained with lead citrate and viewed by Philip CM-120 transmission electron microscopy (Philips, Eindhoven, The Netherlands).

\section{Murine models}

SCID mice (5to 6 weeks old, Shanghai Laboratory Animal Center, Shanghai, China) were injected subcutaneously with $2 \times 10^{7} \mathrm{DB}$ cells into the flank. In the murine model established with DB cells transfected with PCYT1A shRNA or negative control (CON shRNA, five mice each group), tumor volume was calculated as $0.5 \times a$ (length) $\times b$ (width) $)^{2}$ since the date of formation (day 0). In murine model established with DB cells, treatments
(10 mice per group) were started once tumor reached about $0.5 \mathrm{~cm} \times 0.5 \mathrm{~cm}$ in surface (day 0 ). The untreated group received dimethyl sulfoxide, whereas the treated group received BBR once daily for 14 days ( $5 \mathrm{mg} / \mathrm{kg} / \mathrm{day}$ ). Mice were allocated to different groups randomly. Animals were used according to protocols approved by the Shanghai Rui Jin Hospital Animal Care and Use Committee.

\section{Micro positron emission tomography/computed tomography} imaging

Mice were subjected to positron emission tomography/computed tomography analysis 1 week after treatment. Positron emission tomography/computed tomography imaging was performed on an Inveon MM 
Platform (Siemens Preclinical Solutions, Knoxville, TN, USA) as previously reported. ${ }^{14}$ Radio tracer ${ }^{11} \mathrm{C}$-Choline was synthesized and injected to anesthetized mice through the tail vein. ${ }^{23}$ Mean standardized uptake values were determined as dividing the relevant regions of interest concentration by the ratio of the injected activity to the body weight.

\section{Statistical analysis}

Two-tailed Student's $t$-test was applied to compare two groups with similar variance. Association between different factors or parameters was determined by $X^{2}$-test. $P<0.05$ was considered significant. Values were presented as mean \pm s.e.m. Assays were set up in triplicate unless otherwise noted in the Figure legends. Statistical analyses were performed on SPSS 13.0 software (IBM Corp., Armonk, NY, USA).

\section{RESULTS}

MYC was overexpressed in DLBCL and induced aberrant choline metabolism

As revealed by immunohistochemistry, MYC was highly expressed (MYC staining $\geqslant 40 \%)^{22}$ in $44 \%(47 / 108)$ of DLBCL patients (Figure 1a) and significantly correlated with extranodal involvement and an International Prognostic Index, indicating
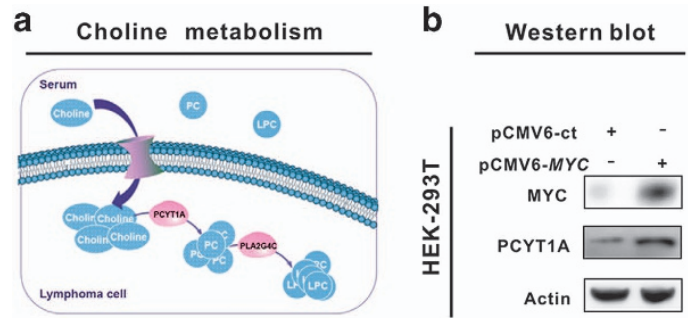

C

ChIP assay
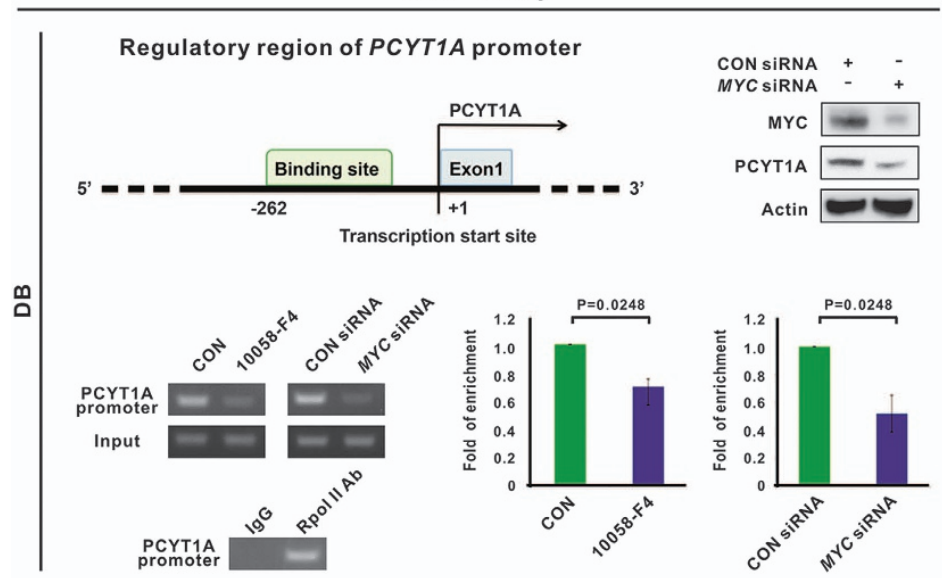

d

Luciferase assay
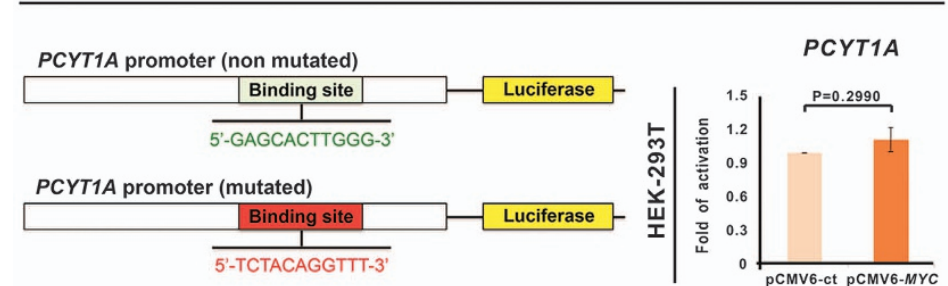

f

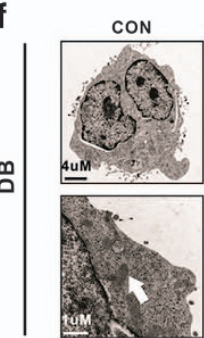

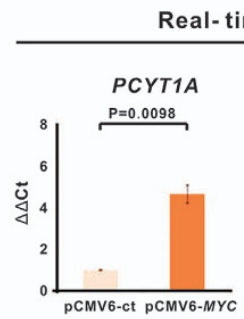
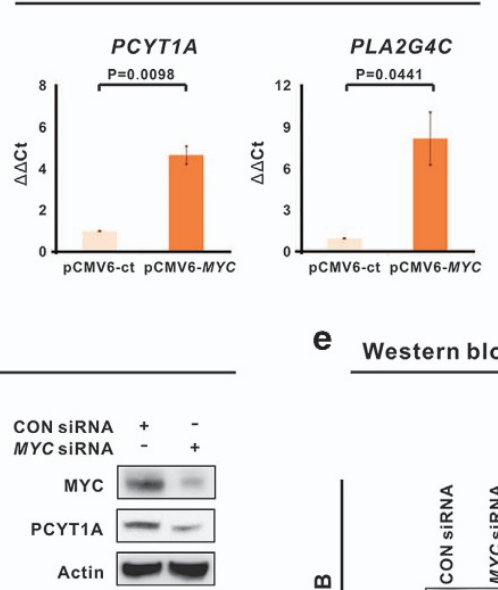

e

Western blot

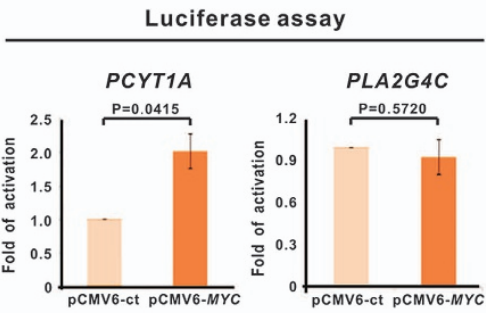

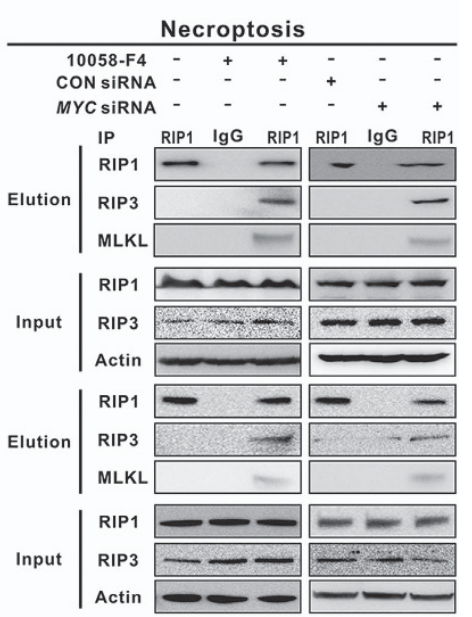
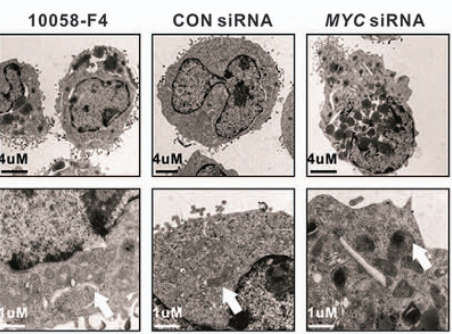

Figure 2. MYC acted on lymphoma choline metabolism by regulating PCYT1A expression. (a) Schematic description of choline metabolism. PCYT1A, phosphate cytidylyltransferase 1 choline- $\alpha$; PLA2G4C, phospholipase A; PC, phosphatidylcholine; LPC, lysophoaphatidylcholine. (b) HEK-293T cells were transfected with pCMV6-MYC plasmid or control plasmid (pCMV6-ct). The efficiency of transfection was confirmed by western blot (left panel). Expression of PCYT1A and PLA2G4C were assessed by quantitative real-time PCR (middle panel). HEK-293T cells were transfected with promoter-driven luciferase reporter of PCYT1A and PLA2G4C. Luciferase reporter activity was measured at $24 \mathrm{~h}$ after transfection (right panel). (c) Schematic diagram represented the regulatory region of PCYT1A promoter (upper panel). The activity of MYC binding to the PCYT1A promoter was measured by chromatin immunoprecipitation assay in DB cells either treated with 10058 -F4 (40 $\mu \mathrm{M})$ for $48 \mathrm{~h}$ or transfected with MYC siRNA. DNA-protein complexes from DB cells were precipitated with anti-MYC antibody and amplified with primers amplifying MYC binding sites on PCYT1A promoter using PCR and quantitative real-time PCR (lower panel). Antibody against RNA Polymerase II was referred as positive control. Nonspecific IgG was referred as negative control. (d) Schematic diagram represented the PCYT1A promoter reporters with or without mutation of MYC binding site (left panel). HEK-293T cells were transfected with mutated promoter-driven luciferase reporter of PCYT1A. Luciferase reporter activity was measured at $24 \mathrm{~h}$ after transfection (right panel). (e) Co-immunoprecipitation showed increased formation of RIP1/RIP3/MLKL complex in DB and Ramos cells, either treated with $10058-\mathrm{F} 4$ (DB, $40 \mu \mathrm{m}$; Ramos, $30 \mu \mathrm{M}$ ) for $48 \mathrm{~h}$ or transfected with MYC siRNA. (f) Transmission electron microscopy showed typical necroptotic cells after 10058-F4 treatment or MYC siRNA transfection. Data in $\mathbf{b}, \mathbf{c}$ and $\mathbf{d}$ were represented as mean \pm s.e.m. Assays in $\mathbf{b}, \mathbf{c}$ and $\mathbf{d}$ were set up in triplicate. 
intermediate-high or high risk (Supplementary Table S1). Patients were subsequently divided into two groups according to MYC expression: MYC-High (MYC staining $\geqslant 40 \%$ ) and MYC-Low (MYC staining $<40 \%$ ).

Serum samples obtained from 30 de novo DLBCL cases (15 each for MYC-High and MYC-Low group, Supplementary Table S3) were analyzed by UPLC-quadruple/time-of-flight mass spectrometry. Representative total ion chromatography was shown in Supplementary Figure S1A. Based on 2708 features obtained after normalization, including 787 spectral features for ESI+ and 1921 spectral features for ESI-, principle component analysis score plots showed a separating trend between MYC-High, MYC-Low groups and repeated quality controls (Figure $1 \mathrm{~b}$, left panel). OPLSDA models revealed satisfactory modeling and predictive ability with one predictive component and one orthogonal component for both $E S I+(R 2 Y=0.86, Q 2=0.5)$ and $E S I-(R 2 Y=0.886$, $\mathrm{Q} 2=0.592$, Figure $1 \mathrm{~b}$, middle panel), as validated by response permutation test (Figure $1 \mathrm{~b}$, right panel).

A total of 47 significantly altered serum metabolites with variable importance for projection $>1$ in the above-mentioned OPLS-DA models and Student's $t$-test $P$-value $<0.05$ between MYC-High and MYC-Low group were identified (Supplementary Table S2). These metabolites were further performed for the enrichment of KEGG pathways using ConsensusPathDB (http://cpdb.molgen.mpg.de), resulting in choline metabolism in cancer (KEGG hsa05231) and glycerophospholipid metabolism pathway (KEGG hsa00564) with the highest significance (Figure 1c). Meanwhile, gene expression profile of frozen tumor tissue samples from nine non-GCB DLBCL cases (Supplementary Table S4) confirmed that MYC-High DLBCL had a distinct gene pattern of cancerous choline metabolism and glycerophospholipid metabolism pathway (Figure 1d, left panel). Choline metabolism-related genes PCYT1A, PLA2G4C and PDGFC, as well as glycerophosphoslipid metabolism-related genes PCYT1A, PLA2G4C, ACHE, PHOSPHO1 and LPGAT2 were identified by Student's $t$-test with $P$-value $<0.05$ (Figure $1 \mathrm{~d}$, right panel). This association between MYC overexpression and aberrant choline metabolism was then confirmed by serum metabolomics assay in a validation set of 50 DLBCL cases (25 each for MYC-High and MYC-Low group, Supplementary Table S3), as revealed by OPLS-DA models $(\mathrm{R} 2 \mathrm{Y}=0.957, \mathrm{Q} 2=0.549$ for $\mathrm{ESI}+\mathrm{R} 2 \mathrm{Y}=0.931$, $\mathrm{Q} 2=0.491$ for $\mathrm{ESI}-$, Figure 1e).

To elucidate the regulatory mechanism of MYC on lymphoma choline metabolism, cellular levels of choline, PC(16:0/22:6), LPC (16:0) and LPC(18:0) were assessed by UPLC-triple quadrupole mass spectrometry. Compared with untreated or negative control (CON siRNA-transfected) cells, 10058-F4-treated or MYC siRNAtransfected B-lymphoma cells displayed a significant decrease in cellular levels of choline and its phosphorylated derivatives (Figure 1f and Supplementary Figure S1B).

Together, these data indicated that MYC overexpression is related to aberrant choline metabolism.

MYC dysregulated B-lymphoma choline metabolism by transcriptional activation of PCYT1A

As schematically summarized in Figure $2 a$, choline is catalyzed to phosphatidylcholine (PC), which is regulated by PCYT1A. Downstream metabolites of PC mainly include lysophoaphatidyl choline (LPC), which is regulated by phospholipase A2 (PLA2, encoded by PLA2G4C). When HEK-293T cells were transfected with pCMV6-MYC plasmid (Figure 2b, left panel), ectopic expression of MYC resulted in remarkably higher levels of PCYT1A and PLA2G4C (main regulatory enzymes) than those of vehicletransfected cells (pCMV6-ct, Figure 2b, middle panel). To identify the key enzyme directly regulated by MYC, luciferase reporter assay was performed on HEK-293T cells and regions of promoter from human genomic DNA were cloned into a dual luciferase reporter vector GV238. The results showed that MYC enhances the gene and protein expression of PCYT1A (Figure 2b). Putative potential conserved MYC binding site within the regulatory region of $P C Y T 1 A$ promoter were predicted using JASPAR database (http://jaspar.genereg.net/cgi-bin/jaspar_db.pl). Chromatin immunoprecipitation assay was conducted in DB cells to verify the physiological interaction of MYC with promoter region of PCYT1A. MYC was able to bind with PCYT1A and the binding activity declined upon treatment with 10058-F4 or transfection with MYC siRNA (Figure 2c). Moreover, when luciferase reporter assay was performed on HEK-293T cells with MYC binding site mutation, the transcriptional activity of PCYT1A was no longer altered by MYC overexpression (Figure $2 \mathrm{~d}$ ).

To gain insight into the biological role of MYC-induced aberrant choline metabolism in B-cell lymphoma, DB and Ramos cells were either treated with MYC inhibitor 10058-F4 or transfected with MYC siRNA (Figure 2e, left panel). The results showed that both pharmaceutical and molecular inhibition of MYC induce B-lymphoma cell necroptosis, as revealed by co-immunoprecipitation with increased binding activity between RIP1, RIP3, and specific downstream mediator of necroptosis MLKL (RIP1/RIP3/MLKL complex, Figure 2e, right panel). ${ }^{24}$ Meanwhile, lymphoma cell necroptosis (swelling mitochondria and intact nuclei membrane) was further confirmed by transmission electron microscopy (Figure 2f).

Therefore, through transcriptional activation of PCYT1A, MYC induced aberrant choline metabolism in B-lymphoma cells, resulting in an increased expression of PCYT1 $A$, the major isoform of choline phosphatecytidylyl transferase required for phosphatidylcholine synthesis and an inhibition of B-lymphoma cell necroptosis.

PCYT1A modulated MYC-mediated B-lymphoma cell necroptosis in a mitophagy-dependent manner and was related to disease progression in DLBCL

To determine the functional consequence of MYC-induced PCYT1A activation on DLBCL, B-lymphoma cells were transfected with siRNA targeting PCYT1A (PCYT1A siRNA). As confirmed by western blot, molecular silencing of $P C Y T 1 A$ resulted in decreased PCYT1A expression, whereas MYC remained constant (Figure $3 a$ ). Compared with negative control (CON siRNA), PCYT1A siRNAtransfected cells exhibited remarkable growth inhibition (Figure $3 b$ ) and necroptosis induction (Figure $3 c$ and Supplementary Figure S2A). Mitophagy is a selective form of macro-autophagy in which mitochondrial are selectively degraded in autophagy lysosomes and functions as a tumor suppressor. ${ }^{25}$ Molecular silencing of PCYT1A increased mitophagy, as evaluated by co-immunofluorescence of LC3A/B with mitochondrial (MTC, Figure $3 \mathrm{~d}$ and Supplementary Figure S2B). Meanwhile, B-lymphoma cell necroptosis was reversed by an addition of mitophagy inhibitor Mdivi-1 (Figure 3c, upper panel and Supplementary Figure S1A), suggesting that PCYT1A induced necroptosis in a mitophagy-dependent manner.

Clinically, PCYT1A mRNA was significantly increased in tumor samples of 108 patients with DLBCL, as compared to reactive hyperplasia (Figure 3e). The patients with PCYT1A expression level over and equal to the median value 2.335 were regarded as PCYT1A-High group, whereas those below the median value were included in PCYT1A-Low group. Increased MYC positivity was more frequently observed in PCYT1A-High group than in PCYT1A-Low group (Figure 3f). Accordingly, representing as the main metabolite of choline metabolic pathway, serum PC (16:0/22:6) level was significantly lower in PCYT1A-High group than in PCYT1A-Low group (Figure $3 \mathrm{~g}$ ). PCYT1A overexpression was also associated with an International Prognostic Index, indicating intermediate-high and high-risk (Supplementary Table S1). 
a
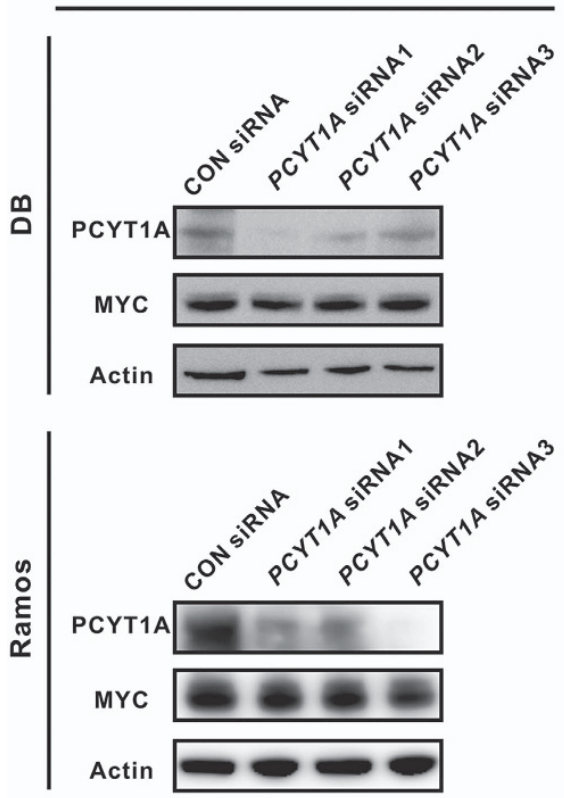

b
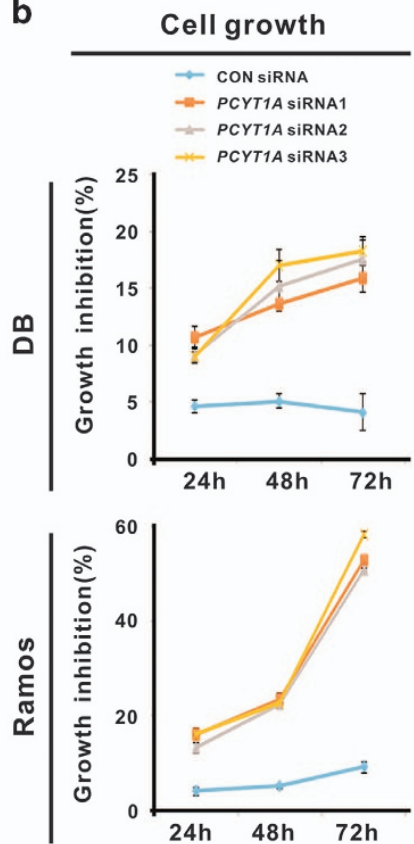

C

\begin{tabular}{ccccc} 
Necroptosis & & \\
\hline CON SiRNA & + & - & - & - \\
PCYT1A siRnA1 & - & + & + & + \\
Mdivi-1 & - & - & - & + \\
IP & RIP1 & IgG & RIP1 & RIP1
\end{tabular}

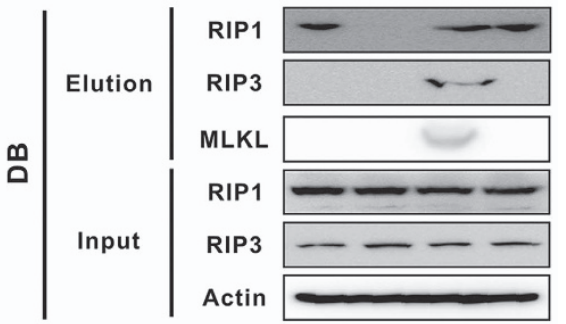

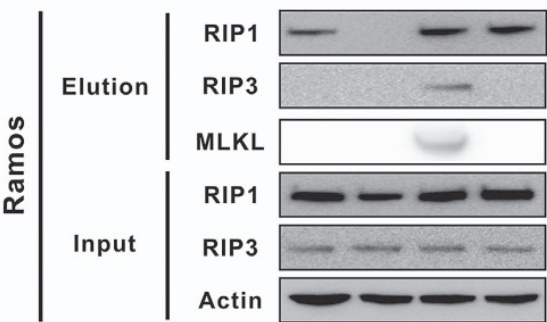

d

Mitophagy
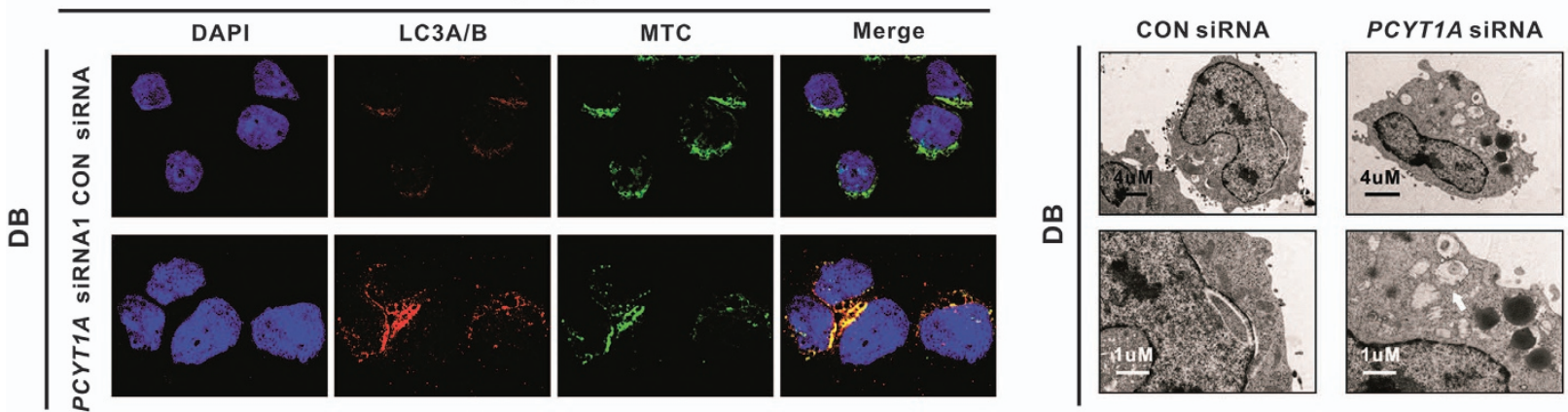

e

Real-time PCR
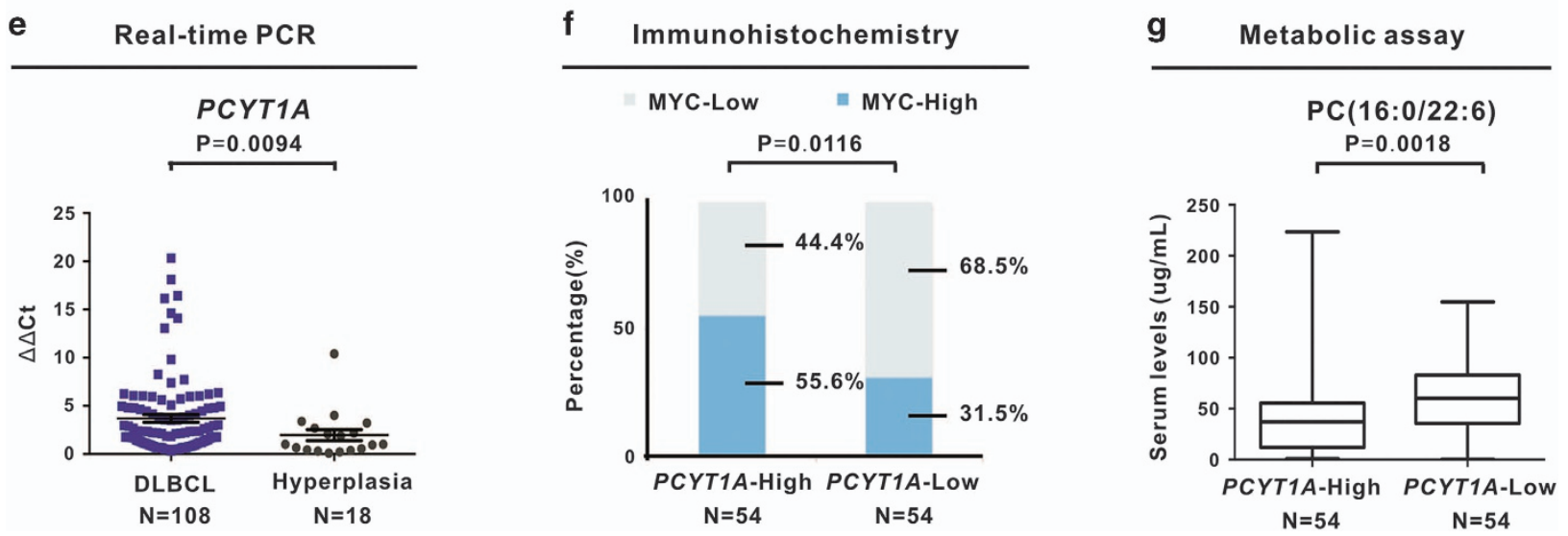

Figure 3. PCYT1A was related to mitophagy-dependent necroptosis and disease progression in DLBCL. (a) Protein levels of PCYT1A and MYC in DB and Ramos cells transfected with PCYT1A siRNA or CON siRNA were assessed by western blottig. (b, c) Cell growth (b) and necroptosis (c) were detected in cells transfected with PCYT1A siRNA or treated with mitophagy inhibitor Mdivi-1 (50 $\mu \mathrm{m})$. (d) Representative immunofluorescence images of LC3A/B (red) and mitochondrial (MTC, green) in DB cells transfected with PCYT1A siRNA1 or CON siRNA. Cells were counterstained with 4',6-diamidino-2-phenylindole (DAPI) (blue). (e) PCYT1A gene expression was assessed by quantitative real-time PCR in DLBCL $(n=108)$ and reactive hyperplasia $(n=20)$. (f, $\mathbf{g})$ Tumor MYC expression (f) and serum PC(16:0/22:6) level $(\mathbf{g})$ were calculated according to PCYT1A expression in DLBCL. Data in $\mathbf{b}$, e and $\mathbf{g}$ were represented as mean \pm s.e.m. Assay in $\mathbf{b}$ was set up in triplicate. 

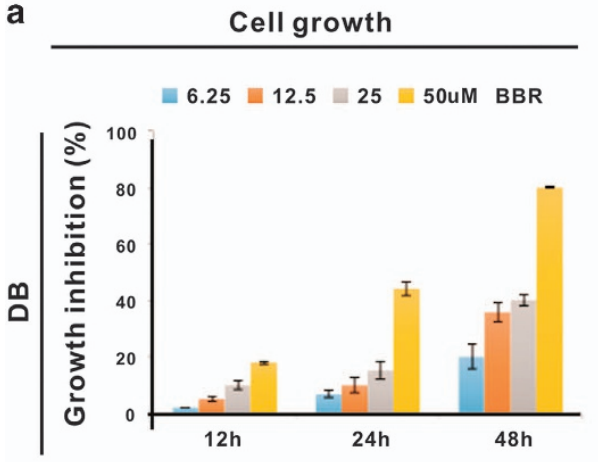

W $6.25=12.5=25=50 \mathrm{uM}$ BBR

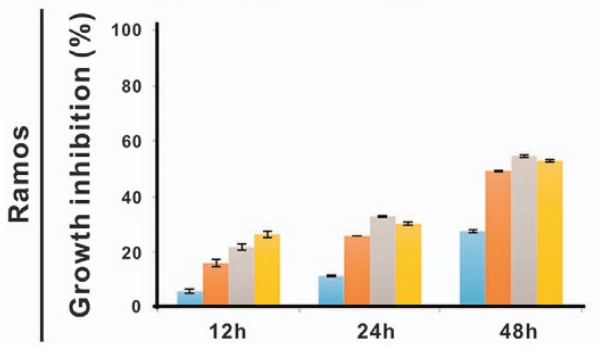

b
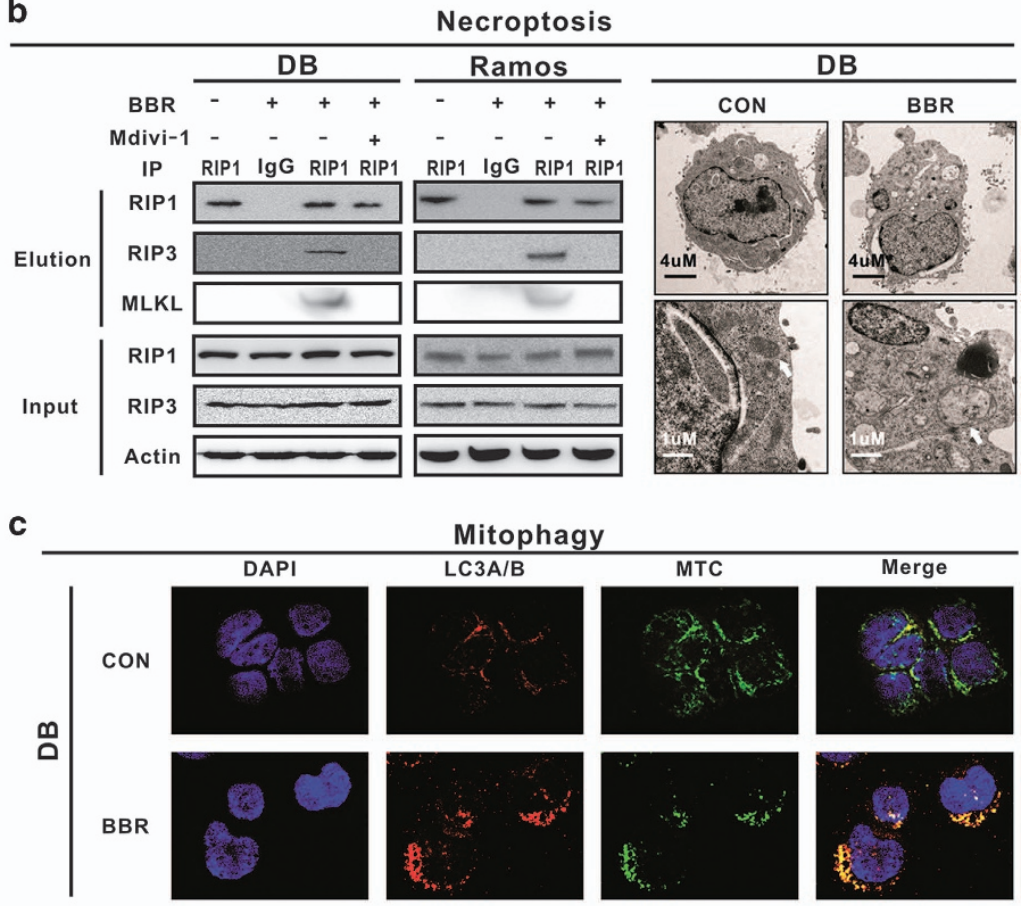

e Luciferase assay

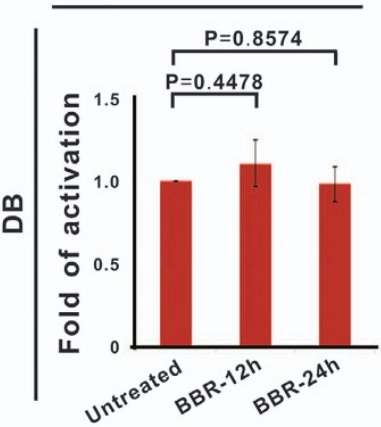

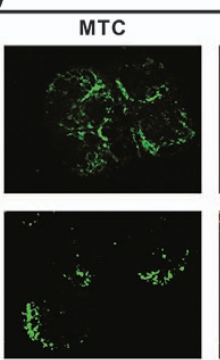

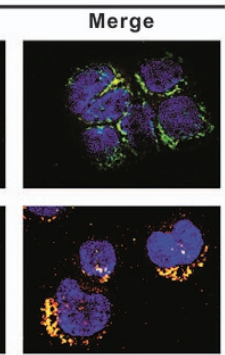

Real-time PCR

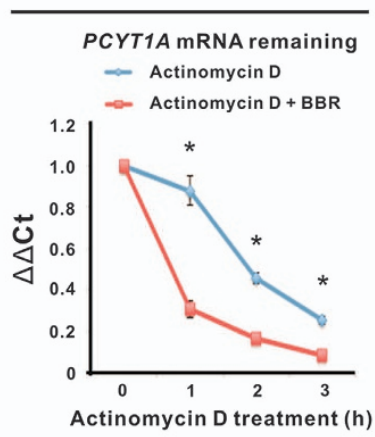

d
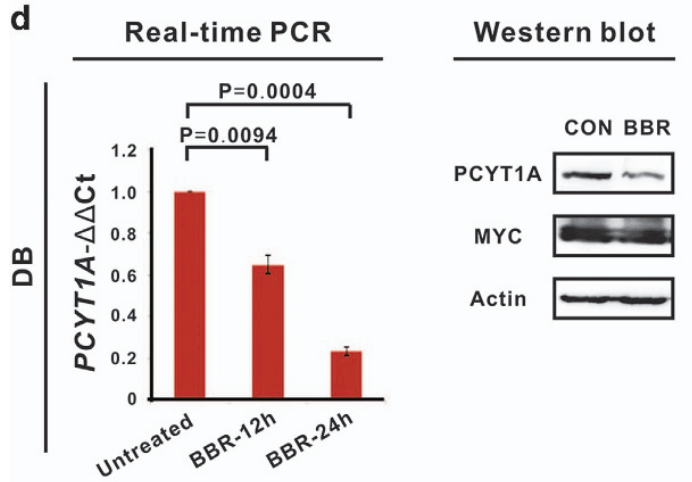

f

Western blot

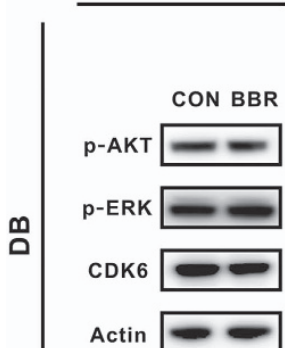

Cell growth inhibition

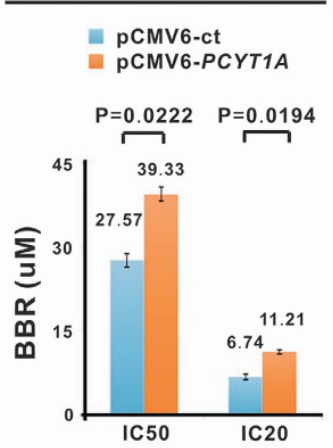

g

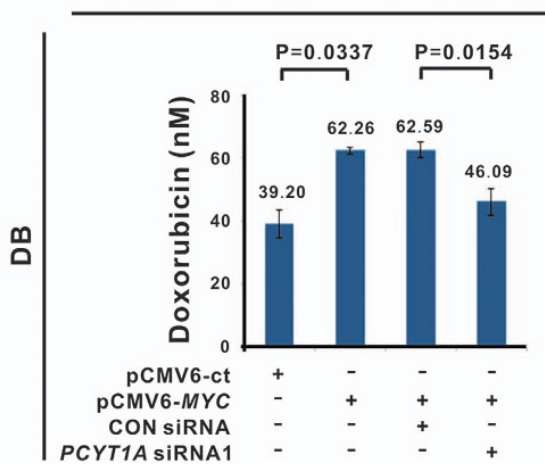

Figure 4. Therapeutic targeting of PCYT1A by berberine (BBR) in B-lymphoma cells. (a) Growth inhibition was measured in DB and Ramos cells treated with BBR. (b, c) Necroptosis (b) and mitophagy (c) were detected in BBR-treated cells (30 $\mu \mathrm{m}$ and $20 \mu \mathrm{m}$, respectively) for $48 \mathrm{~h}$. (d) PCYT1A was assessed in DB cells by quantitative real-time PCR and western blotting. (e) PCYT1A promoter plasmid and pRL-TK were cotransfected into DB cells. BBR $(30 \mu \mathrm{m})$ was added to cells $12 \mathrm{~h}$ or $24 \mathrm{~h}$ before cell lysis. Cells were collected and firefly and Renilla luciferase activity were measured (left panel). Actinomycin D $(5 \mu \mathrm{g} / \mathrm{ml})$ was added to DB cells for different intervals, either alone or treated with BBR $(30 \mu \mathrm{m})$ for $24 \mathrm{~h}$ (right panel). (f) $\mathrm{p}$-AKT, p-ERK and CDK6 were assessed in DB cells by western blot (left panel). Comparing with the pCMV6-Cttransfected cells, IC50 and IC20 of BBR were increased in DB cells transfected with pCMV6-PCYT1A (right panel). (g) Comparing with the pCMV6-ct-transfected cells, IC50 of doxorubicin, but not BBR, was increased in DB cells transfected with pCMV6-MYC. MYC-induced increase of IC50 was decreased by PCYT1A siRNA, as compared with negative control (CON siRNA). Data in $\mathbf{a}, \mathbf{d}, \mathbf{e}, \mathbf{f}$ and $\mathbf{g}$ were represented as mean \pm s.e. $\mathrm{m}$. Assays in $\mathbf{a}, \mathbf{d}, \mathbf{e}, \mathbf{f}$ and $\mathbf{g}$ were set up in triplicate. 
a
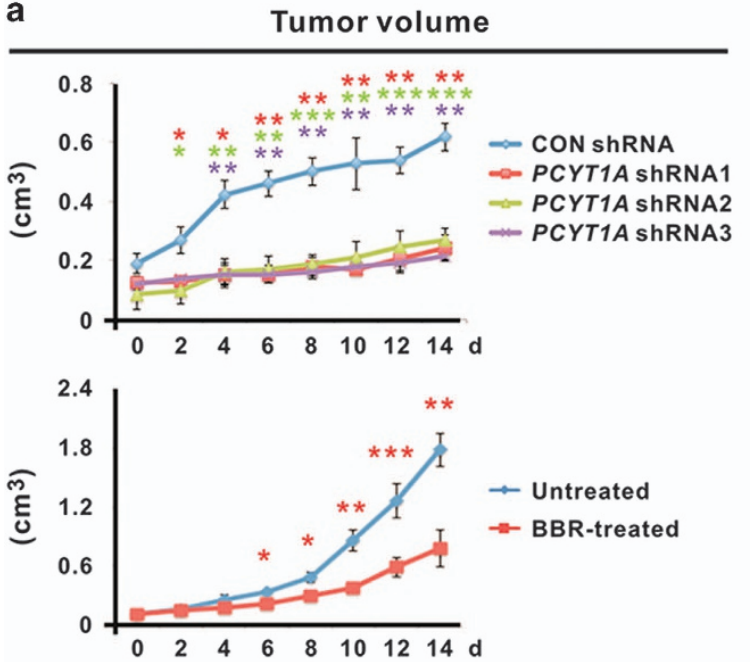

c
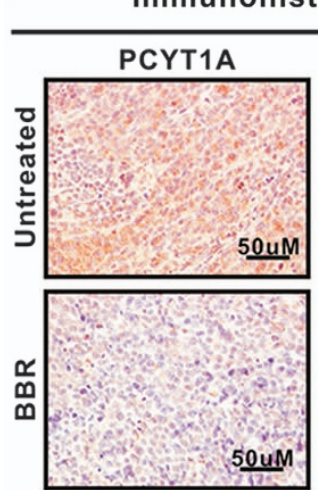

e

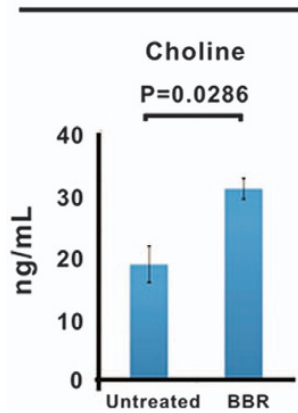

- Untreated

- BBR-treated
MYC
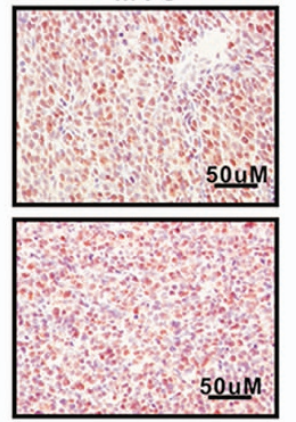

Metabolic assay

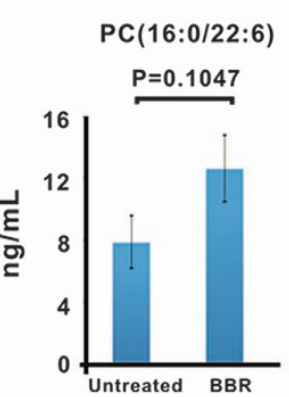

b

11C-Choline microPET/CT

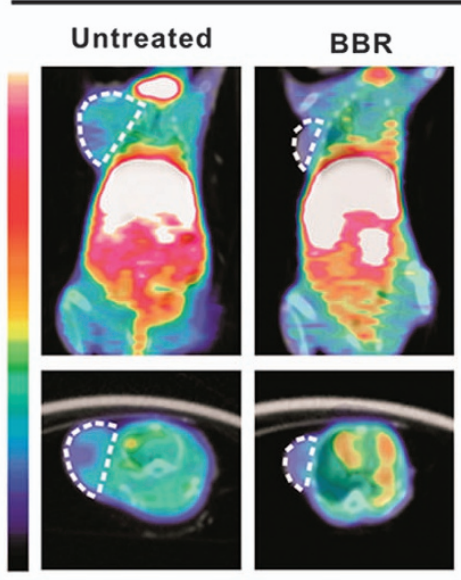

d Transmission electron microscopy
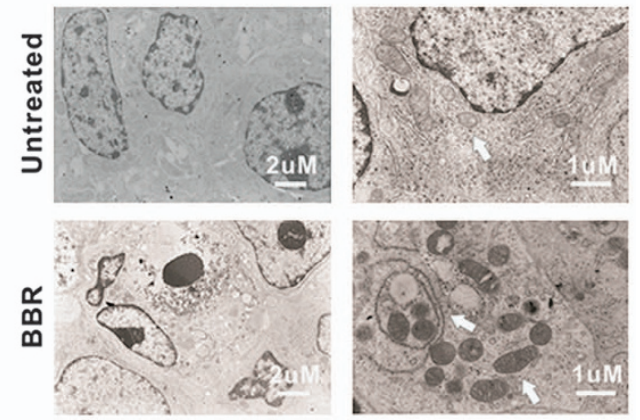
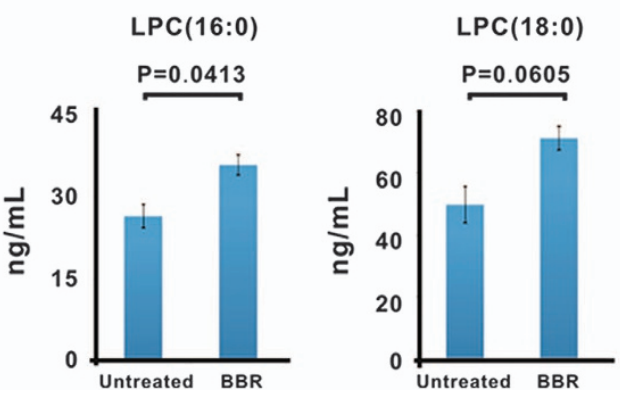

Figure 5. In vivo activity of BBR on murine xenograft B-lymphoma model. (a) Tumor size of xenograft B-cell lymphoma with subcutaneous injection of DB cells transfected with PCYT1A shRNA or control shRNA (CON shRNA, upper panel, $N=5$ for each group). Tumor size was measured in BBR group and the untreated group in xenograft B-cell lymphoma with subcutaneous injection of DB cells (lower panel, $N=10$ for each group). ${ }^{* * *} P<0.001,{ }^{* *} P<0.01$ and ${ }^{*} P<0.05$ comparing with the untreated group. (b) ${ }^{11} \mathrm{C}$-Choline micro-positron emission tomography/computed tomography was performed one week after BBR treatment and standardized uptake value (SUV) intensity was observed. (c) PCYT1A and MYC expression were detected by immunohistochemistry. (d) Necroptosis and mitophagy were observed under transmission electron microscopy. (e) Serum levels of choline and its phosphorylated derivatives were measured by UPLC-triple quadrupole mass spectrometry. Data in a and e were represented as mean \pm s.e.m. Assay in e was set up in five mice.

Therapeutic targeting of PCYT1A by BBR counteracted

MYC-induced B-lymphoma cell growth via mitophagy-dependent necroptosis

At a clinically achievable concentration, ${ }^{17}$ lipid-lowering alkaloid BBR inhibited B-lymphoma cell growth in a time- and dosedependent manner (Figure 4a), consistent with the induction of necroptosis and the presence of mitophagy (Figures $4 \mathrm{~b}$ and $\mathrm{c}$ ). The gene and protein expression of PCYT1A were significantly downregulated by $B B R$, while MYC protein remained constant (Figure 4d). To clarify how BBR targeted PCYT1A, PCYT1A promoter activity was analyzed by transfecting DB cells with the PCYT1A promoter reporter plasmid. No significant difference in reporter activity was found between the untreated group and the BBR group (Figure $4 \mathrm{e}$, left panel), prompting us to examine the possibility that BBR treatment may affect the stability of PCYT1A mRNA, resulting in lower expression levels of PCYT1A. The stability of PCYT1A mRNA was then estimated by measuring mRNA levels 
after treatment with Actinomycin $D(5 \mu \mathrm{g} / \mathrm{ml})$, a nucleic acid synthesis inhibitor, for the indicated times by quantitative realtime PCR. The results showed that BBR decreases the transcript stability and promotes the degradation of PCYT1A mRNA (Figure $4 \mathrm{e}$, right panel). Although BBR could downregulate a series of kinases, including PI3K/AKT, MAPK, and CDKs, ${ }^{26,27}$ these changes were not obvious in MYC-overexpressing DB cells (Figure 4f, left panel). Of note, resistance to BBR was observed in B-lymphoma cells transfected with pCMV6-PCYT1A, as compared to pCMV6-ct (Figure 4f, right panel), which confirmed that the downregulation of PCYT1A is a key event in BBR-induced cell growth inhibition. Moreover, compared to vehicle-transfected cells (pCMV6-ct), ectopic expression of MYC (pCMV6-MYC) induced significantly increased IC50 of DB cells towards chemotherapeutic agents like doxorubicin, cisplatin and gemcitabine (but not BBR). In these MYC-overexpressing cells, molecular silencing of PCYT1A sensitized cells to these chemotherapeutic agents (Figure $4 \mathrm{~g}$ and Supplementary Figure S3).

BBR exhibited in vivo anti-lymphoma activity and induced in situ necroptosis.

In the murine model established with subcutaneous injection of PCYT1A shRNA-transfected DB cells or negative control (CON shRNA), the size of xenograft tumors in the PCYT1A shRNA groups were significantly smaller than those of the CON shRNA group (Figure 5a, upper panel). Accordingly, in the murine model established with subcutaneous injection of DB cells, the size of xenograft tumors in the BBR group were significantly smaller than those of the untreated group after 6 days of treatment (Figure $5 \mathrm{a}$, lower panel). ${ }^{11}$ C-Choline micro-positron emission tomography/ computed tomography was then carried out to visualize choline metabolism of tumors a week following the treatment. Compared with those in untreated mice, standardized uptake value intensity of tumors was significantly reduced in the BBR-treated mice (Figure 5b). As in vitro study, PCYT1 A expression was decreased in the tumors of the BBR group, without any obvious change in the MYC protein (Figure 5c). To search for more evidence of in situ tumor cell necroptosis, ultrastructural study was performed on mice tumor. Typical cell necroptosis (swelling mitochondria and intact nuclei membrane) and mitophagy (increased mitochondrial density and accumulation of mitochondria within the doublemembrane autophagy lysosomes) ${ }^{14,25}$ were frequently observed in BBR-treated tumors (Figure 5d). Accordingly, serum levels of choline, as well as its phosphorylated derivatives $P C(16: 0 / 22: 6)$, $\operatorname{LPC}(16: 0)$ and $\operatorname{LPC}(18: 0)$ were also restored by BBR treatment (Figure 5e).

These results provided in vivo evidence that progression of MYC-driven lymphoma can be tackled by altering downstream effector of choline metabolism with lipid-lowering agents.

\section{DISCUSSION}

Growing evidence suggest that the activation of oncogenes can reprogram tumor cell metabolism. ${ }^{28}$ MYC is a key oncogene and critically involved in lipid synthesis, including those of cholesterol, fatty acid and glycerophospholipid. ${ }^{12,29,30}$ Here we support a direct link between MYC overexpression and dysregulated choline metabolism (a major component of glycerophospholipids). These data in turn highlight the pivotal role of oncogenic MYC on lipid metabolism.

PCYT1A is the major isoform for the key enzyme CTP (choline phosphate cytidylyltransferase), essential for PC synthesis during lipid metabolism. In neuroblastoma, MYC modulates lipid synthesis by coordinating with MondoA. ${ }^{29}$ As for lung cancer, MYC upregulates cytosolic phospholipase A2 and increases phosphatidylinositols and arachidonate-containing phospholipids, which are required for tumor cell survival and proliferation. ${ }^{31}$ In the present study, PCYT1A was upregulated by MYC and contributed to dysregulated choline metabolic pathways, suggesting an alternative mechanism involved in MYC-mediated lymphoma cell metabolism and tumor progression. This is consistent with experimental findings in intestinal epithelial, which shows that PCYT1A determines malignant transformation of their normal counterparts. $^{32}$

Choline and its phosphorylated derivatives are implicated in the initiation and execution of necroptosis, an alternative caspaseindependent cell death by modulating RIP1/RIP3 complex, also known as necrosome. ${ }^{33}$ Alterations in lipid metabolism may damage cellular and subcellular membrane, cause imbalance between mitochondrial fusion and fission, initiate mitophagy and lead to necroptosis. Therefore, PCYT1A may not only act as a downstream effector of MYC on regulation of choline metabolism, but also a biomarker of MYC-mediated lymphoma cell necroptosis and lymphoma progression in DLBCL.

It is promising to treat oncogene-driven tumors through targeting downstream cell metabolism. For example, MYCmediated glutamine metabolism could be modulated by glutaminase-specific inhibitor, which diminishes tumorigenesis and prolongs the survival of the mice with MYC-associated hepatocellular carcinoma. ${ }^{34}$ Furthermore, inhibition of fatty acid oxidation has recently been proposed as a potential treatment for triple-negative breast cancer with MYC overexpression. ${ }^{35}$ BBR is a quartenary ammonium salt belonging to the proto-berberine group of isoquinolone alkaloids and extracted from Chinese herbs known for its diverse pharmacological properties, notably the lipid-lowering effect. ${ }^{16}$ The effect of BBR on mitochondrial fragmentation and depolarization has also been revealed ${ }^{36}$ as contributing to necroptotic cell death in cancer cells. ${ }^{37} \mathrm{We}$ showed that BBR inhibits B-lymphoma cell growth by accelerating mRNA degradation of PCYT1A and inducing mitophagydependent necroptosis, suggesting an alternative therapeutic relevance of BBR on MYC-High lymphoma. In addition, necroptosis may arise from chemotherapy treatment, accounting for the cell death observed in apoptosis-defective tumor cells. ${ }^{38}$ Induction of necroptosis to bypass apoptosis and to overcome chemoresistance has been achieved in hematological malignancies, such as acute myeloid leukemia and MLL-rearranged acute lymphoblastic leukemia. ${ }^{39-41}$ Consistently, our data further opened up promising treatment avenues for BBR to reverse the chemoresistant effect of MYC by affecting MYC-downstream effectors.

In conclusion, MYC positively regulated PCYT1A expression and was responsible for the dysregulation of choline metabolism in DLBCL. These metabolic alterations could be reversed by lipidlowering agent BBR, providing a clinical rationale of lipid-lowering strategy in treating MYC-high lymphoma.

\section{CONFLICT OF INTEREST}

The authors declare no conflict of interest.

\section{ACKNOWLEDGEMENTS}

This study was supported, in part, by research funding from the National Natural Science Foundation of China (81325003, 81520108003, 81670716 and 81201863), the Shanghai Commission of Science and Technology (14430723400, 14140903100 and 16JC1405800), the National Key Research and Development Program (2016YFC0902800), Shanghai Municipal Education Commission Gaofeng Clinical Medicine Grant Support (20152206 and 20152208), Multi-center Clinical Research Project by Shanghai Jiao Tong University School of Medicine (DLY201601), SMC-Chen Xing Scholars Program, Chang Jiang Scholars Program, Innovation Fund Projects of Shanghai Jiao Tong University (BXJ201607), Collaborative Innovation Center of Systems Biomedicine and the Samuel Waxman Cancer Research Foundation. 


\section{AUTHOR CONTRIBUTIONS}

WLZ and SJC designed research studies. JX conducted the experiments, LW, XCF and CFW performed pathological analysis. XFJ and BL analyzed data. ZZ and $Y Z$ provided reagents. WLZ, RPG and AJ wrote the manuscript.

\section{REFERENCES}

1 Pasqualucci L, Dalla-Favera R. SnapShot: diffuse large B cell lymphoma. Cancer Cell 2014; 25: 132-132 e131.

2 Younes A. Prognostic significance of diffuse large B-cell lymphoma cell of origin: seeing the forest and the trees. J Clin Oncol 2015; 33: 2835-2836.

3 Crump M, Leppa S, Fayad L, Lee JJ, Di Rocco A, Ogura M et al. Randomized, double-blind, phase III trial of enzastaurin versus placebo in patients achieving remission after first-line therapy for high-risk diffuse large B-cell lymphoma. J Clin Oncol 2016; 34: 2484-2492.

4 Taub R, Kirsch I, Morton C, Lenoir G, Swan D, Tronick S et al. Translocation of the c-myc gene into the immunoglobulin heavy chain locus in human Burkitt lymphoma and murine plasmacytoma cells. Proc Natl Acad Sci USA 1982; 79: 7837-7841.

5 Horn H, Ziepert M, Wartenberg M, Staiger AM, Barth TF, Bernd HW et al. Different biological risk factors in young poor-prognosis and elderly patients with diffuse large B-cell lymphoma. Leukemia 2015; 29: 1564-1570.

6 Lwin T, Zhao X, Cheng F, Zhang X, Huang A, Shah B et al. A microenvironmentmediated c-Myc/miR-548m/HDAC6 amplification loop in non-Hodgkin B cell lymphomas. J Clin Invest 2013; 123: 4612-4626.

7 Cottereau AS, Lanic H, Mareschal S, Meignan M, Vera P, Tilly H et al. Molecular profile and FDG-PET/CT total metabolic tumor volume improve risk classification at diagnosis for patients with diffuse large B-cell lymphoma. Clin Cancer Res 2016; 22: 3801-3809.

8 McKeown MR, Bradner JE. Therapeutic strategies to inhibit MYC. Cold Spring Harb Perspect Med 2014; 4, pii: a014266.

9 Dang CV. MYC on the path to cancer. Cell 2012; 149: 22-35.

10 Sarkozy C, Traverse-Glehen A, Coiffier B. Double-hit and double-proteinexpression lymphomas: aggressive and refractory lymphomas. Lancet Oncol 2015; 16: e555-e567.

11 Benjamin DI, Cravatt BF, Nomura DK. Global profiling strategies for mapping dysregulated metabolic pathways in cancer. Cell Metab 2012; 16: 565-577.

12 Dang CV. MYC, metabolism, cell growth, and tumorigenesis. Cold Spring Harb Perspect Med 2013; 3, pii: a014217.

13 Eberlin LS, Gabay M, Fan AC, Gouw AM, Tibshirani RJ, Felsher DW et al. Alteration of the lipid profile in lymphomas induced by MYC overexpression. Proc Natl Acad Sci USA 2014; 111: 10450-10455.

14 Xiong J, Bian J, Wang L, Zhou JY, Wang Y, Zhao Y et al. Dysregulated choline metabolism in T-cell lymphoma: role of choline kinase-alpha and therapeutic targeting. Blood Cancer J 2015; 5: 287.

15 Shalapour S, Karin M. Fatty acid-induced T cell loss greases liver carcinogenesis. Cell Metab 2016; 23: 759-761.

16 Kong W, Wei J, Abidi P, Lin M, Inaba S, Li C et al. Berberine is a novel cholesterollowering drug working through a unique mechanism distinct from statins. Nat Med 2004; 10: 1344-1351.

17 Zhang Y, Li X, Zou D, Liu W, Yang J, Zhu N et al. Treatment of type 2 diabetes and dyslipidemia with the natural plant alkaloid berberine. J Clin Endocrinol Metab 2008; 93: 2559-2565.

18 Casey SC, Amedei A, Aquilano K, Azmi AS, Benencia F, Bhakta D et al. Cancer prevention and therapy through the modulation of the tumor microenvironment. Semin Cancer Biol 2015; 35(Suppl): S199-S223.

19 Wang L, Liu L, Shi Y, Cao H, Chaturvedi R, Calcutt MW et al. Berberine induces caspase-independent cell death in colon tumor cells through activation of apoptosis-inducing factor. PLoS ONE 2012; 7: e36418.

20 Piccaluga PP, Fuligni F, De Leo A, Bertuzzi C, Rossi M, Bacci F et al. Molecular profiling improves classification and prognostication of nodal peripheral T-cell lymphomas: results of a phase III diagnostic accuracy study. J Clin Oncol 2013; 31: 3019-3025.

21 Zheng Z, Cheng S, Wu W, Wang L, Zhao Y, Shen Y et al. c-FLIP is involved in tumor progression of peripheral T-cell lymphoma and targeted by histone deacetylase inhibitors. H Hematol Oncol 2014; 7: 88.

22 Maura F, Guidetti A, Pellegrinelli A, Dodero A, Pennisi M, Caprioli C et al. Highdose chemotherapy followed by autologous transplantation may overcome the poor prognosis of diffuse large B-cell lymphoma patients with MYC/BCL2 co-expression. Blood Cancer J 2016; 6: e491.
23 Hara T, Yuasa M. Automated synthesis of [11C]choline, a positron-emitting tracer for tumor imaging. Appl Radiat Isot 1999; 50: 531-533.

24 Zhang J, Yang Y, He W, Sun L. Necrosome core machinery: MLKL. Cell Mol Life Sci 2016; 73: 2153-2163.

25 Chourasia AH, Tracy K, Frankenberger C, Boland ML, Sharifi MN, Drake LE et al. Mitophagy defects arising from BNip3 loss promote mammary tumor progression to metastasis. EMBO Rep 2015; 16: 1145-1163.

26 Liu B, Wang G, Yang J, Pan X, Yang Z, Zang L. Berberine inhibits human hepatoma cell invasion without cytotoxicity in healthy hepatocytes. PLOS ONE 2011; 6: e21416.

27 Mantena SK, Sharma SD, Katiyar SK. Berberine, a natural product, induces G1-phase cell cycle arrest and caspase-3-dependent apoptosis in human prostate carcinoma cells. Mol Cancer Ther 2006; 5: 296-308.

28 Boroughs LK, DeBerardinis RJ. Metabolic pathways promoting cancer cell survival and growth. Nat Cell Biol 2015; 17: 351-359.

29 Carroll PA, Diolaiti D, McFerrin L, Gu H, Djukovic D, Du J et al. Deregulated Myc requires MondoA/Mlx for metabolic reprogramming and tumorigenesis. Cancer Cell 2015; 27: 271-285.

30 Priolo C, Pyne S, Rose J, Regan ER, Zadra G, Photopoulos C et al. AKT1 and MYC induce distinctive metabolic fingerprints in human prostate cancer. Cancer Res 2014; 74: 7198-7204.

31 Hall Z, Ament Z, Wilson CH, Burkhart DL, Ashmore T, Koulman A et al. Myc expression drives aberrant lipid metabolism in lung cancer. Cancer Res 2016; 76: 4608-4618.

32 Arsenault DJ, Yoo BH, Rosen KV, Ridgway ND. ras-Induced up-regulation of CTP: phosphocholine cytidylyltransferase alpha contributes to malignant transformation of intestinal epithelial cells. J Biol Chem 2013; 288: 633-643.

33 Magtanong L, Ko PJ, Dixon SJ. Emerging roles for lipids in non-apoptotic cell death. Cell Death Differ 2016; 23: 1099-1109.

34 Xiang Y, Stine ZE, Xia J, Lu Y, O'Connor RS, Altman BJ et al. Targeted inhibition of tumor-specific glutaminase diminishes cell-autonomous tumorigenesis. J Clin Invest 2015; 125: 2293-2306.

35 Camarda R, Zhou AY, Kohnz RA, Balakrishnan S, Mahieu C, Anderton B et al. Inhibition of fatty acid oxidation as a therapy for MYC-overexpressing triplenegative breast cancer. Nat Med 2016; 22: 427-432.

36 Pereira GC, Branco AF, Matos JA, Pereira SL, Parke D, Perkins EL et al. Mitochondrially targeted effects of berberine [Natural Yellow 18, 5,6-dihydro-9,10dimethoxybenzo(g)-1,3-benzodioxolo(5,6-a) quinolizinium] on K1735-M2 mouse melanoma cells: comparison with direct effects on isolated mitochondrial fractions. J Pharmacol Exp Ther 2007; 323: 636-649.

37 Zhang LY, Wu YL, Gao XH, Guo F. Mitochondrial protein cyclophilin-Dmediated programmed necrosis attributes to berberine-induced cytotoxicity in cultured prostate cancer cells. Biochem Biophys Res Commun 2014; 450: 697-703.

38 Long JS, Ryan KM. New frontiers in promoting tumour cell death: targeting apoptosis, necroptosis and autophagy. Oncogene 2012; 31: 5045-5060.

39 Bonapace L, Bornhauser BC, Schmitz M, Cario G, Ziegler U, Niggli FK et al. Induction of autophagy-dependent necroptosis is required for childhood acute lymphoblastic leukemia cells to overcome glucocorticoid resistance. J Clin Invest 2010; 120: 1310-1323.

40 Steinhart L, Belz K, Fulda S. Smac mimetic and demethylating agents synergistically trigger cell death in acute myeloid leukemia cells and overcome apoptosis resistance by inducing necroptosis. Cell Death Dis 2013; 4: e802.

41 Urtishak KA, Edwards AY, Wang LS, Hudome A, Robinson BW, Barrett JS et al. Potent obatoclax cytotoxicity and activation of triple death mode killing across infant acute lymphoblastic leukemia. Blood 2013; 121: 2689-2703.

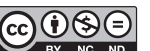

This work is licensed under a Creative Commons AttributionNonCommercial-NoDerivs 4.0 International License. The images or other third party material in this article are included in the article's Creative Commons license, unless indicated otherwise in the credit line; if the material is not included under the Creative Commons license, users will need to obtain permission from the license holder to reproduce the material. To view a copy of this license, visit http:// creativecommons.org/licenses/by-nc-nd/4.0/

(c) The Author(s) 2017

Supplementary Information accompanies this paper on Blood Cancer Journal website (http://www.nature.com/bcj) 